\title{
A Heterogeneous Information Processing System Based on Smart City
}

\author{
Yong Li \\ Beijing University of Technology \\ Beijing, China
}

\begin{abstract}
Solution to solve heterogeneous information process sing and related technology integration in Internet of Thing is proposed. A platform based on the technology is designed, to achieve the integration of information, expression and push of integration information, achieve a decentralized unified expression of application data, realize the external system interfaces and networking applications, interaction interface between the Internet and the client application, achieve the data expression and push in the Internet of Things.
\end{abstract}

Keywords-Smart city; information processing system; unified expression

\section{INTRODUCTION}

Things heterogeneous expression of the common technical processing technology and heterogeneous information of the original information was studied, proposed to solve things in heterogeneous information related processing technology and expression techniques. The use of these technical design of heterogeneous information processing, sharing common expression system platform architecture, detailed in the application of information fusion platform, the integration of information, expression and push to achieve a unified expression of decentralized application data to achieve the external General expression and interaction between the system and the Internet of Things application interface, Internet application interfaces and client interface to achieve the data on the Internet of Things expression and push.

With the development of the Internet of Things, Things applications in data processing issues become very prominent, become a hot topic[1][2][3]. Furthermore, the Internet of Things applications require real-time networking, accurate, low power consumption and efficient processing of data, but the existing data processing mode for now difficult to meet these requirements[4][5][6].

Faced with heterogeneous information for things, the need to solve the key technology of heterogeneous networking information processing: information fusion technology and expression; expression and the formation of a common standard interface for heterogeneous information and data networking. Things heterogeneous information including original generic expression, standardized data interface networking, unified communications capabilities and common IT capabilities open standard interface; for our networking with multiple data sources, heterogeneous and other characteristics, proposed to support unified communications capabilities and open platform architecture common IT capabilities, build a multi-service environment things mass

\author{
Yang Li \\ Digital China (China)Limited \\ Beijing, China
}

information platform and the ability to support multi-service networking platform, and utility applications to achieve universal networking platform demonstration in support of multi-service networking platform and performance.

\section{SYSTEM ARCHITECTURE}

To achieve the heterogeneous data processing and fusion expression, design heterogeneous data processing and information is essential to the expression of fusion protein architecture. Focus on resolving the current data are independent of Internet of Things, the information can not understand each other's problems, architecture system research Internet of Things fusion expression data types, and heterogeneous data storage and processing research, information operations under Internet of Things.

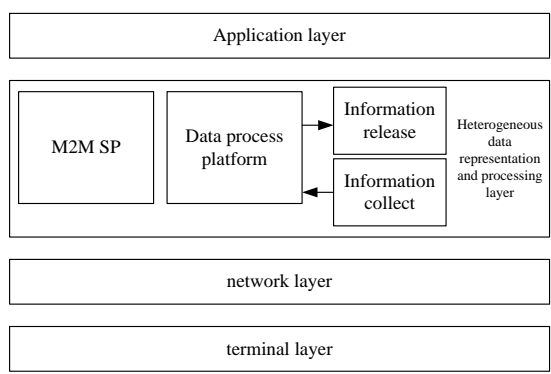

FIGURE I . SYSTEM ARCHITECTURE.

Unified expression and processing of heterogeneous data fusion architecture is divided into four layers: the terminal layer, network layer, expression and processing of heterogeneous data layer, application layer.

Terminal layer: M2M terminals, gateways, etc., is the basis for data processing and integration of heterogeneous information sources expression.

Network Layer: Provides network transport and the ability to provide for heterogeneous information processing and fusion expression.

Heterogeneous data representation and processing layer: the underlying data fusion protein and processed by an enhanced platform M2MSP Things, heterogeneous data processing platform to share information publishing platform.

Application layer: Internet of Things subscribers and users of information.

M2MSP Platform: The platform after the introduction of information operations in Internet of things, need to increase 
the basic data collection module, by incorporating this module and terminal, and this module with the network, combined with the needs of business, complete collection and analysis of data.

Heterogeneous Data Processing Platform: The platform is completed to collect basic data of networked heterogeneous data object information stored in the processing, ontology modeling and identification data fusion expression of things to share with the transaction, the terminal and the terminal of the agreement, and eventually based on information stored data mining and information-based operational information.

Information sharing processing platform: The platform interoperability complete networking and mobile Internet, data processing heterogeneous data processing platform, microblogging can be published through the machine and machine facial makeup. Microblogging machine, machine mask underlying behavior of the data that can be collected to heterogeneous data integration platform for further processing.

\section{INFORM HETEROGENEOUS DATA STORAGE AND PROCESS}

Now, the enterprise data warehouse and relational database adept at handling structured data, and can store large amounts of data, but expensive. This kind of data requirements limit the types of data that can be handled, and this disadvantage also affects inertia brought into the data warehouse in face of massive heterogeneous data for quick exploration, this usually means that valuable data source has never been excavated within the organization.

NoSQL and semi-structured data storage are the key to large heterogeneous data integration solutions, data storage needs with key / value storage model, to solve the too more fields, integration difficult.

Presently, data management architecture and parallel are considered, solving scale high performance requirements of concurrent data access and data storage, which is a mature technology. The understanding of unstructured data, is key to achieve large data resources, knowledge, but there are still no substantive breakthrough and progress, which is an immature technology.

Therefore, this article uses data directly expressed as information, semantic-based data storage is the key to the next big data networking information search solutions: data must be converted into information storage, follow-up treatment, this information as the basis for aggregation, search, mining, push and so on.

Heterogeneous data need to regulate the expression of a template for information technology: terminal register to the platform at first, must provide two template as description:

\footnotetext{
- Information element data Templates: in the form of xslt

Action elementdata template: terminal according to their behavior acceptable
}

\section{A. Information Collection Interface}

In Internet of things, there are a lot of heterogeneous information and data, such as structured data, semi-structured data. Information gathering interface usedfordata collected from the following dimensions: from M2MSP platform to collect data related to the application of the relevant data, terminal data and networking network. From the information publishing platform to collect information and data of things.

\section{B. Information Release}

The information related to the Internet of Things, by machine or machine microblogging Facebook, etc., published on the Internet and mobile publishing platform interoperability.

\section{Data Market}

Internet of Things information data mart is operating trading market, the need to trade and the need for the operator to complete the data ordering, transfer, payment and other related interfaces.

\section{Design Principles of Interface}

Design principles of interface are as follows:

- The design of Data expressiontemplate according to specification, such as XSL.

- If the machine subscriptions, data passed can be raw format, rather than the express format.

Data upload and express the process:

- Terminal upload data and resolve orignal data

- Platform record orignal template data

- terminal officially reported data

- According to the data interpretation by platform, display data to user in understandable format

Machine user subscription and data transfer processes:

- machine users to subscribe

- Platform Send explain template data

- The machine user record template data

- The machine user gets a new data (user-readable form, but the raw data reported by the terminal)

- The machine user interpretation template data according to the data, resolve the data, and further processing

\section{CONCLUSION}

Solution to solve heterogeneous informationprocessingand related technology integration in Internet of Thing is proposed. A platform based on the technology is designed, to achievethe integration of information,expression and push of integration information, achieve a decentralized unified expression of application data, realize the external system interfaces and networking applications, interaction interface between the Internet and the client application, achieve the data expression and push in the Internet of Things. 


\section{ACKNOWLEDGEMENTS}

This work was financially supported by the project2012AA01A403.

\section{REFERENCES}

[1] Li Hao ;Xue Lei ; Zhu Yan ; The application and implementation research of smart city in China,System Science and Engineering (ICSSE), 2012 International Conference on, 2012, Page(s): 288 $-292$.

[2] Xiaoguo, Yu ; Chuang, Fan, Ecological Analysis of Smart City Based on the Numerical Simulation Technology, Intelligent Systems Design and Engineering Applications (ISDEA), 2014 Fifth International Conference on, 2014 , Page(s): 438- 441.

[3] Roscia, M. ; Longo, M. ; Lazaroiu, G.C, Smart City by multi-agent systems, Renewable Energy Research and Applications (ICRERA), 2013 International Conference on, 2013, Page(s): 371 - 376.

[4] Veeckman, C.; van der Graaf, S.The city as living labortory: A playground for the innovative development of smart city applications, Engineering, Technology and Innovation (ICE), 2014 International ICE Conference on, 2014, Page(s): 1-10.

[5] Ulligan, C.E.A.; Olsson, M. Architectural implications of smart city business models: an evolutionary perspective, Communications Magazine, IEEE, Volume:51, Issue:6, 2013, Page(s): 80- 85.

[6] Kehua Su ; Jie Li ; Hongbo Fu, Smart city and the applications, Electronics, Communications andControl (ICECC), 2011 International Conference on, 2011 , Page(s): 1028- 1031 